Cipango Cahiers d'études japonaises

$21 \mid 2014$

Nouveaux regards sur les arts de la scène japonais II

\title{
La journée classique de kagura de Hayachine
}

Interprétations savantes et réalités

A Classic Day of Hayachine Kagura: Scholarly Interpretations and the Reality of the Performance

\section{Catherine Delpuech}

\section{(2) OpenEdition Journals}

\section{Édition électronique}

URL : https://journals.openedition.org/cipango/2205

DOI : 10.4000/cipango.2205

ISSN : 2260-7706

\section{Éditeur}

INALCO

Édition imprimée

Date de publication : 31 décembre 2014

ISSN : 1164-5857

\section{Référence électronique}

Catherine Delpuech, « La journée classique de kagura de Hayachine », Cipango [En ligne], 21 | 2014, mis en ligne le 08 septembre 2016, consulté le 30 juin 2021. URL : http://journals.openedition.org/ cipango/2205; DOI : https://doi.org/10.4000/cipango.2205

\section{(c) (†) (5)}

Cipango est mis à disposition selon les termes de la Licence Creative Commons Attribution - Pas d'Utilisation Commerciale 4.0 International. 


\title{
La journée classique de kagura de Hayachine Interprétations savantes et réalités
}

\author{
A Classic Day of Hayachine Kagura: Scholarly \\ Interpretations and the Reality of the Performance
}

Catherine Delpuech Docteur en ethnologie et sociologie comparative

\section{Introduction}

À quelques $500 \mathrm{~km}$ de Tōkyō, dans le Japon du Nord-Est (Tōhoku), plus précisément le département d'Iwate, deux hameaux, en apparence tranquilles, voire désertés sous le manteau de neige de l'hiver, se livrent pourtant une bataille séculaire à propos de leur tradition de « divertissements divins » kagura 神楽. 
56 Cahiers d'études japonaises $n^{\circ} 21$

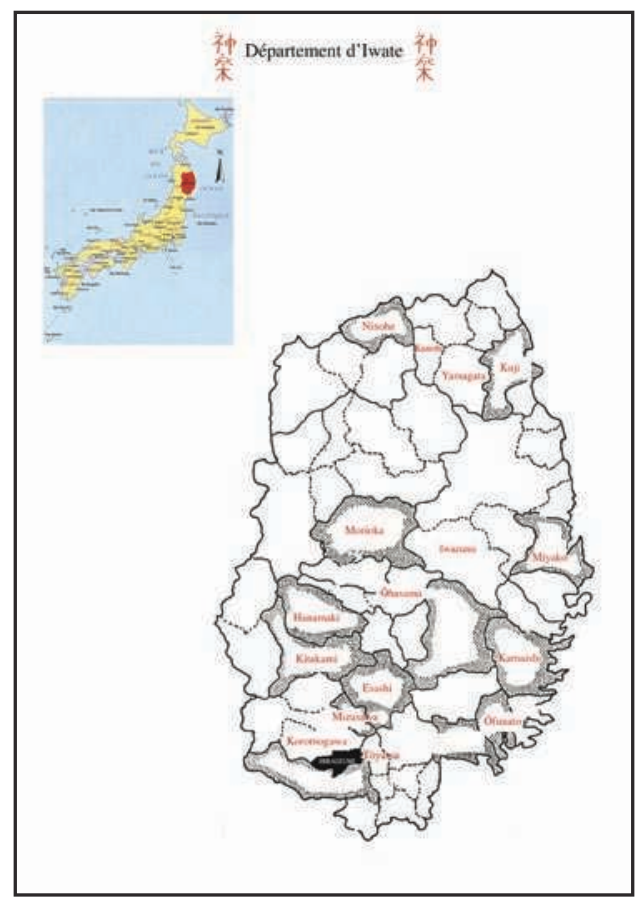

FIGURE 1.

Carte du département d'Iwate.

Source : carte dessinée par Catherine Delpuech

De la capitale, on parvient à Take 岳 et Ōtsugunai 大償 au bout de quelques heures du shinkansen Yamabiko, puis en autocar et enfin en taxi, à moins d'être attendu par une connaissance. Jusque dans les années 1950, il n’y avait pas de route et on accédait à Take, le village le plus reculé accroché au flanc sud du mont Hayachine, à cheval ou en carriole. Environ mille mètres plus bas, à quinze kilomètres de distance, Ōtsugunai repose au pied de la montagne. Les deux hameaux se trouvent donc dans une relation d'opposition, mais aussi de complémentarité, irréversiblement liée à un contexte géophysique précis : un Japon montagnard profond (Take) et un Japon rural ouvert dans la vallée (Ōtsugunai). De cette partition issue des conditions naturelles, les habitants ont élaboré un système complexe s'ap- 
pliquant à tous les niveaux : historique, religieux, géographique, social et même stylistique de la danse et de la musique de leurs kagura. De l'avis des danseurs, elle aurait en effet instauré des attitudes corporelles différentes. Cette systématisation a incontestablement été influencée par les religieux à l'origine des courants de kagura perpétués dans les deux villages depuis au moins cinq cents ans.

Formalisé sous la dénomination a-un 阿云, motivée et construite par un tissu de correspondances liées au bouddhisme ésotérique, ce système allie et oppose un principe féminin $a$, associé à Ōtsugunai, à un principe masculin un, rattaché au village de Take. Ainsi la forme des réceptacles (ou corps) divins shintai 身体 des sanctuaires respectifs implique l'aspect $a$ ou un des divinités tutélaires dénommées gongen 権 現 des sanctuaires respectifs. Le réceptacle de celui d'Ōtsugunai étant une pierre ovale, le kagura et son style sont reconnus comme féminins. Du côté de Take, la montagne, aisément identifiable au phallus, tient lieu de shintai, le style de kagura y est par conséquent qualifié de masculin. En réalité, peu importe que les attributs mâles ou femelles existent ou non - la fameuse pierre d'Ōtsugunai a disparu, elle aurait été volée! Peu importe aussi que le style soit réellement viril ou féminin, ce qui compte est l'existence d'une dualité dont les habitants sont fermement convaincus, bien qu'ils ignorent dans leur majorité les arguments savants qui la fondent. Les habitants justifient ainsi l'existence de deux courants de kagura, kagura-ryzu 神楽流, et leurs singularités, faisant de la tradition un enjeu constant de rivalités et la rendant identitaire. Ceci explique, pour une part à mon sens, sa vigueur actuelle.

La relation avec le sacré passe évidemment par le rapport mythico-historique avec le territoire national et local, les croyances autochtones, la symbolique religieuse et, de plus, par l'expérience corporelle. On glisse ainsi implicitement du corps divin aux techniques du corps dansant qui semblent indissociables des représentations mentales auxquelles « le sentiment de nature », habituellement attaché à la japonité, donne lieu.

\section{Définition des kagura}

Pour les Japonais connaisseurs de théâtre, les divertissements divins relèvent des « arts folkloriques » geino $\overline{0}$ 芸能. Ils en considèrent certains aspects comme représentatifs des danses autochtones les plus anciennes. Kagura 神楽 désigne effectivement les danses et musiques du culte shintô ou du moins du fond de 


\section{CIPANGO}

58 Cahiers d'études japonaises nº 21

croyances locales amalgamées au shintô. Il est en effet probable que l'organisation du culte soit apparue bien après les premiers kagura. Plusieurs combinaisons de lecture des deux sinogrammes composent ce mot, mais toujours le premier signifie « dieux », le second « réjouissances » et spécialement la danse et la musique. Le sens global coïncide avec la réalité de la tradition de la région de Hayachine étudiée ici et l'image que les villageois en ont, soit des divertissements joués pour et par les dieux, à la fois pour leur plaisir et celui des hommes. L'ethnologue Honda Yasuji 本田安次 signale la première occurrence du terme kagura dans le Man.yoshy 万葉集 ${ }^{1}$, mais il écrit :

Les caractères étaient lus sasa, probablement en relation avec le son des clochettes suzu, tenues par les desservantes de temples, les miko, au cours de leur kagura. Les danseuses agitaient les feuilles de bambou dont le bruissement, sayasaya, prévenait les dieux de l'imminence d'un kagura et les convoquait ${ }^{2}$.

Sasa est aussi le nom d'un bambou nain dont une branche servit d'instrument rituel à la déesse Ame no Uzume lors de sa danse mythique de l'ouverture de la caverne que beaucoup d'amateurs de ce théâtre et d'érudits japonais entendent comme le kagura des origines. Toutefois, l'étymologie la plus courante de kagura est celle de place ou siège des dieux, kamu ou kami kura 神座, où le deuxième caractère peut s'écrire différemment et se lit $z a^{3}$; le vocable se serait contracté et palatalisé en kagura. Kami-za signale la présence des dieux qui regardent danser Ame no Uzume, et, concrètement, le za était (et est) l'endroit du temple ou du

1. Le Man.yōsh̄u (Recueil des dix mille feuilles [ou des dix mille générations]) est une grande anthologie qui relate des poèmes très anciens, remontant jusqu'à l'ère de l'empereur Nintoku (313-399). Il a été achevé en 759. D'autres chroniques font référence au kagura, ainsi le Recueil d'histoires anciennes (Kogoshüi 古語拾遺), compilé en 807 par Inbe no Ironari pour compléter le Kojiki, qui mentionne le kagura qui eut lieu pour fêter les divinités terrestres et célestes lors de l'établissement de la capitale de l'empereur Jinmu dont le règne aurait commencé en 660 avant notre ère.

2. Honda Yasuji 本田安次, Minzoku geinō, kagura 民俗芸能 神楽 (Spectacles populaires folkloriques, kagura), Tōkyō, Bunkachō 文化庁, 1970, p. 22.

3. Za signifie « siège, place; s'asseoir ». 
sanctuaire où les artistes locaux jouaient pour honorer les dieux ${ }^{4}$. Par extension, il devint le nom des troupes artistiques villageoises invitées à se produire pour les fêtes religieuses matsuri.

Donnés dans tout le Japon à l'occasion des rites saisonniers ou des rites de la vie humaine, les kagura mettent en scène des mythes, des événements historiques ou représentent des rituels. On en distingue deux catégories : les kagura de Cour ou mi-kagura 御神楽 ( $m i$ est une particule honorifique) et une deuxième forme, rustique, celle des kagura de villages ou sato-kagura 里神楽 5 présentée ici. Selon René Sieffert, ces derniers seraient des variantes champêtres, postérieures de deux à trois siècles aux kagura de Cour des grands temples de Kyōto. Il n'existe cependant aucun moyen de vérifier cette allégation. L'intéressant est que des glissements se produisent d'un modèle à l'autre et que des formes hybrides ont vu le jour, donnant naissance à de multiples mélanges. Les divertissements divins ayant gardé un sens religieux sont encore très vivants, surtout en milieu villageois. Ce sont des danses propitiatoires et de purification supposées éloigner les épidémies, les incendies, assurer longévité, fécondité et bonheur aux hommes et bonnes récoltes. En milieu urbain, les kagura intégrés à des fêtes autrefois religieuses, mais qui souvent ne sont plus que des parades animées, tendent à se folkloriser, devenant alors de simples spectacles.

\section{Particularités des kagura de Take et d'Ōtsugunai}

Les kagura villageois font l'objet de diverses subdivisions. Ceux de Take et d'Ōtsugunai sont généralement reconnus en tant que kagura de lion ou shishi

4. Attachés à un temple ou un sanctuaire, ces $z a$ ou littéralement « lieux de résidence des dieux » (miyaza 宮座), étaient organisés en confréries paroissiales qui s'occupaient de l'aspect culturel des « affaires divines » (shinji 神事), précisément de l'organisation des rites.

5. Paradoxalement, cette expression est parfois usitée pour des kagura célébrés dans les villes (à Tōkyō par exemple). Il ne s'agit donc pas d'une dénomination localisatrice mais typologique. 
kagura 獅子神楽 ${ }^{6}$ caractérisés par l'apparition obligatoire d'un (ou de plusieurs) personnage(s) zoomorphe(s) portant un masque de lion shishi ${ }^{7}$. Parmi les shishi kagura, on distingue les kagura d'ascètes montagnards ou yamabushi kagura 山伏 神座 ${ }^{8}$ auxquels est rattachée la tradition qui nous occupe, nommée ou renommée tradition de Hayachine 早池峰 par Honda Yasuji 本田安次 ${ }^{9}$. Cependant, dans les deux localités, on ne parle pas de shishi mais uniquement de gongen 権現, pour deux raisons : les shishi n'ont pas d'âme, ils n'incarnent rien alors que les masques des gongen sont animés et consacrés au cours d'une cérémonie particulière. Ils

6. Honda Yasuji 本田安次, Nihon no minzoku geinō 日本の民俗芸能 (Spectacles populaires folkloriques du Japon), vol. 5, Tōkyō, Mokujisha 木耳社, 1966.

7. Shishi est un terme générique qui désigne toutes sortes d'animaux sauvages ou surnaturels ayant habituellement pour territoire la montagne. On compte ainsi parmi les shishi des lions chinois karashishi, des léopards hi, des chiens coréens koma inu ou des espèces de dragons (à quatre pattes), voir à ce propos SUGAWARA Seiichirō 菅原盛一 郎, Nihon no geinō, Hayachine-ryū yamabushi kagura 日本之芸能 早地峰流山伏神 楽 (Spectacles populaires japonais, kagura yamabushi, les courants de Hayachine), Iwate, Tōwa-chō kyōiku iinkai 東和町教育委員会, 1979 [1969], p. 182. À Take et Ōtsugunai, les masques ressemblent le plus souvent à ceux de sangliers, de cochons, voire de gros chiens. Â Tonō, on peut voir des masques de daims et de cerfs. Pour plus de détails sur les shishi, voir le numéro 42-44 de la revue Matsuri 祭り (Fêtes).

8. Les yamabushi sont des sorciers-ermites, des mystiques qui jouent le rôle de médecins guérisseurs et de thaumaturges. Ils suivent la discipline rigoureuse de la voie de l'ascèse shugendō, d'où leur autre nom d'adeptes du shugen ou shugenja 修験者 ou encore de pratiquants de l'ascèse, gyōja 行者. La voie de l'ascèse est un mouvement syncrétique qui mêle aux pratiques des écoles bouddhiques ésotériques (Tendai, Shingon, Kegon) des éléments de Taoïsme et de chamanisme, déjà plus ou moins amalgamés au shintô, et le culte de la montagne. Elle vise l'obtention de pouvoirs magiques par l'ascèse, ellemême conditionnée par un apprentissage initiatique et soumise à une loi secrète juhō 哾 法. Le shintô est la voie des kami, soit la voie des dieux ou des « êtres supérieurs » selon l'étymologie populaire. Elle est communément tenue pour antérieure à l'introduction de la culture chinoise et du bouddhisme.

9. D’après les villageois éclairés, le nom de 《kagura de Hayachine 》早池峰神楽 viendrait de Honda. Il vint pour la première fois dans la région en 1931 et œuvra plus tard pour le rapprochement des deux écoles, leur permettant d'obtenir en 1976 la qualification de trésor culturel bunka-zai 文化財 que chacun des villages ne pouvait obtenir seul. C'est ainsi que cette relation se créa, non sans problèmes, de même que le concept de kagura de Hayachine, et étendit à l'époque la réputation de la tradition au plan national. 
matérialisent Seoritsu, divinité tutélaire du mont Hayachine vénérée au sanctuaire de ce village, et, à Ōtsugunai, une trinité divine constituée par le dieu de la lune et fils d'Izanagi, Tsukiyomi no Mikoto, le dieu du grondement du tonnerre ${ }^{10}$, Ō Kaminari no Mikoto, et le dieu du riz (ou du grenier à riz), Ina sōtama ou Inakura no Mikoto. De ce fait, le sanctuaire prend le nom de trois sanctuaires sansha et la divinité tutélaire est appelée grand gongen des trois sanctuaires.

D'autre part, le culte des gongen est au cœur des rituels inhérents à la voie de

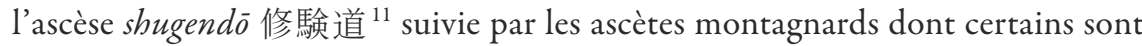
généralement admis comme les fondateurs de la tradition de Take. L'avènement de la divinité Seoritsu 瀬織津, raconté dans la légende de trois sœurs, est conforme aux récits propagés par la voie de l'ascèse en tous lieux où la montagne est sacrée (reizan) au Japon. Ce qui est le cas du mont Hayachine. L'apparition divine entraîne l'élaboration d'un dispositif d'investissement sacré de l'espace par les ascètes qui inventent la tradition en ces lieux. L'école d'Ōtsugunai, bien que rattachée à une autre mouvance religieuse, celle des prêtres Sceau de la Loi, dits hōin, est également marquée par des éléments nettement dérivés des pratiques de la voie de l'ascèse.

La croyance aux gongen est très populaire dans la région : dans presque tous les sanctuaires de villages est enchâssée au moins une tête de gongen, manifestation du dieu local. De plus, quasiment tous les particuliers de Take et d'Ōtsugunai possèdent leur propre gongen, quelquefois deux, comme le danseur Sasaki Hideo 佐々 木秀夫. Ils en parlent avec fierté et respect et les appellent de manière honorifique

10. Ce dieu est très vénéré et beaucoup de sanctuaires, dont celui de Kashima, lui sont consacrés. En général, son réceptacle shintai que l'on voit dans les lieux qui lui sont dédiés est un sabre. De ce fait, les arbres fendus par la foudre sont considérés comme sacrés et il est défendu de les abattre.

11. Le bouddhisme se divise en écoles de pensée exotériques et ésotériques. Ces dernières se caractérisent par une initiation progressive et un abord complexe qui en restreint l'accès aux religieux. Ce sont deux nouvelles conceptions importées de Chine qui se concrétisent au Japon au $\mathrm{IX}^{\mathrm{e}}$ siècle par les écoles de pensée du Tendai et du Shingon, rattachées à la voie de l'ascèse. Pour plus de détails, voir Gaston Renondeau, Le Shugendō : histoire, doctrine et rites des anachorètes dits «Yamabushi », Paris, Imprimerie nationale, 1965; Mryake Hitoshi 宮家準, Shugendō to Nihon shūkyō 修験道と日本宗教 (Le shugendō et la religion japonaise), Tōkyō, Shunjūsha 春秋社, 1996. 
62 Cahiers d'études japonaises $n^{\circ} 21$

gongen-sama. Ichinokura Shun.ichi 一 /倉俊 - ${ }^{12}$, issu d'une famille de danseurs du village d'Ishihatooka (kagura disciple de Take), explique que les dieux et les bouddhas doivent être concrétisés pour devenir visibles aux yeux des hommes ${ }^{13}$. C'est pourquoi on utilise temporairement un masque et une longue robe dont les traits et les motifs respectifs different en fonction du sanctuaire d'appartenance. Les gongen se manifestent de façon omniprésente au cours des rites d'Ōtsugunai et de Take.

Hormis le gongen, les danses de kagura comportent d'autres éléments issus du bouddhisme ésotérique, notamment des éléments de protection contre les mauvais esprits et de magie propitiatoire liés au culte de la montagne, et par conséquent aux pratiques taoïstes et du jugondō coréen ${ }^{14}$, importées du continent vers le VII ${ }^{\mathrm{e}}$ siècle. Elles font explicitement appel à des effets toujours entendus par les habitants comme magiques; ils nomment d'ailleurs certaines d'entre elles danses de prières magiques d'exorcisme kaji kitō-mai 加持祈祷舞. La connaissance des rites anciens dont elles participent était liée au secret et à un enseignement initiatique. Ceci se voit à travers une gestuelle issue des pratiques rituelles des ascètes qui met en action des pas ashibumi 足踏 ou ashigoto 足事 et des sceaux manuels in 印 ou insō 印相 spécifiques. D’autre part, les réceptacles divins que sont les torimono ou objets tenus par les danseurs, l'usage de sabres exorcisants et les costumes sont également propres à ces kagura yamabushi. Enfin, la magie imitative contribue au répertoire. À cet amalgame religieux s'ajoute une complexité chorégraphique, fruit des apports concomitants ou successifs d'expressions théâtrales japonaises ou étrangères de toutes les époques. Ces danses sont donc très hétérogènes.

La tradition de Hayachine ne concerne pas seulement Take et Ōtsugunai. Depuis au moins deux cents ans, leurs maîtres l'ont transmise à beaucoup de troupes des environs sous forme de kagura disciples, les deshi kagura 弟子神楽.

12. ICHINOKURA Shun.ichi一 一倉俊一, Hayachine kagura 早池峰神楽 (Les Kagura de Hayachine), Morioka, Ōhasama-machi kankō kyōkai 大迫町観光協会, 1995, p. 130.

13. La théorie connue sous le nom d' « état originel (les bouddhas) et trace descendue (les dieux) », honji suijaku assimile ces dieux du culte shintô, les kami, à des incarnations ou manifestations provisoires de bouddhas (ou bodhisattvas) dénommées gongen. Dans le contexte shintô, cette théorie se nomme « shintô en partie double » ou ryōbu shintō. L'école de pensée ésotérique japonaise de la Parole Véritable (Shingon) y défend l'idée que chaque dieu peut se manifester sous la forme d'une divinité bouddhique.

14. Équivalent de la voie de l'ascèse en Corée. 
C'est ainsi que dans tout le Japon, des troupes de kagura, encore rattachées à d'innombrables temples et sanctuaires, perpétuent des traditions séculaires similaires. Il existe en effet un fonds national où ces troupes puisent mais la plupart d'entre elles ont en outre leur propre répertoire. Selon les localités, le nombre et la forme des danses varient : là où cette tradition est vive, comme dans nos deux villages, il peut y avoir jusqu'à 50 représentations par an. Ailleurs, elles se limiteront à une ou deux. Le répertoire de Take et Ōtsugunai est en outre très riche, il comprend une soixantaine de danses et beaucoup plus de rôles mais tous ne sont plus joués. Entre 30 et 40 danses environ sont représentées au cours des divers programmes de l'année. Leur narration s'inspire d'histoires puisées dans les mythes shinwa 神話 ou est tirée de récits historiques shiden 史伝, ou encore d'anecdotes édifiantes, les setsuwa 説話. Ces histoires sont relatées dans des livrets, plus ou moins remaniés au cours des ans, que les acteurs utilisent. On peut donc parler indifféremment de danses, de pièces, d'exempla, de saynettes ou simplement de kagura comme le font les habitants.

Quel que soit le courant, la tradition est constituée de danses rituelles simples ou complexes accompagnées de musiques (flutte, tambour, cymbales), de chants divins kami uta 神歌 et de récits katarimono 語物, dont le genre parfois archaïsant les place parmi les danses de tradition immémoriale. Les cérémonies présentent habituellement une structure type commune à la majorité des rituels japonais en trois temps. Elles commencent par des actes de purification comprenant une danse qui prépare la descente et l'accueil des dieux, puis d'autres prières gestuelles leur sont offertes. De nos jours, la troisième phase du renvoi des dieux kami okuri 神 送 n'existe plus, cependant les rituels sont quelquefois clos par un repas, le naorai 直会 ${ }^{15}$, où les acteurs retrouvent les autres villageois, des amis et souvent quelques spectateurs invités à la fête. Cela fait partie des modes de réjouissances qui accompagnent les divertissements divins.

\section{La journée classique de kagura : structure et composition}

Au sein du répertoire, les érudits se réfèrent à un ensemble particulier composé de

15. Littéralement « réunion de réparation, de correction 》 de toutes les choses « détestables » avec une connotation taboue. 
12 danses ou pièces ${ }^{16}$ qui constituerait pour eux un rituel complet et spécifique de la tradition de Hayachine. Je qualifie ce rituel de « journée classique » par opposition aux rituels actuellement « courants ». Cette journée classique de kagura est divisée en deux séries dites de l'endroit et de l'envers et composées chacune de 6 danses cérémonielles shiki mai 式舞, nommées ainsi par les connaisseurs de cette tradition. Les érudits locaux considèrent que les kagura de Hayachine sont les seuls à présenter cette structure endroit-envers. Il faut entendre par « Hayachine » une zone relativement vaste qui s'étend dans un périmètre d'au moins $60 \mathrm{~km}$ autour de la ville d'Ōhasama ${ }^{17}$, et non pas au sens strict de « tradition de Hayachine » réservé aux villages de Take et d'Ōtsugunai. En effet, j'ai assisté à de très nombreuses représentations de kagura régionaux, toutes celles jouées par les troupes des alentours ont ce double aspect endroit-envers. Ce qui est logique puisque certaines d'entre elles sont des troupes disciples de l'une ou de l'autre école ou, au contraire, seraient à l'origine de ces deux courants.

Voici comment se déroule la journée classique : les trois premières danses de l'endroit sont la danse des oiseaux Torimai 鳥舞, la danse du vieillard Okina 翁 et, littéralement, celle du troisième vieillard Sanbasō 三番叟, souvent abrégé en Sanba. En principe, Sanbasō danse en troisième, (sanban, d'où son nom), mais il se présente souvent en deuxième position, notamment lors des fêtes d'été. Ce choix répond à la qualité des spectateurs. Il est fait pour que les touristes ne relâchent pas leur attention après la danse des oiseaux, relativement monotone mais obligatoire car c'est à la fois un rite de purification et une convocation des dieux qui vont

16. Les danses de kagura de Hayachine relèvent indéniablement d'un genre théâtral, c'est pourquoi j'utilise alternativement les termes de danses, pièces, voire d'actes pour les cérémonies composées de plusieurs danses.

17. La ville d'Ōhasama, située à $5 \mathrm{~km}$ d'Ōtsugunai et à $15 \mathrm{~km}$ de Take, est un passage obligé pour accéder aux deux villages. Elle est peuplée de 7120 âmes. Au-dessous de ce nombre, tout est qualifié de village mura ou de hameau buraku. Les villages et leurs terres forment des cantons gun, divisés en districts, les me. Le canton de Hienuki inclut quatre districts, Kanegamori, Ōhasama, Uchikawa-me et Sotokawa-me. Bien que les noms des deux derniers signifient respectivement proche de la rivière et loin de la rivière, il ne s'agit pas de la même (rivière kawa). En effet, la rivière Nakaigawa traverse Sotokawame alors que c'est la Takegawa qui parcourt Uchikawame. Uchikawame est découpé en neuf subdivisions $k u$ réparties en deux groupes de villages qui incluent Take et Ōtsugunai. 
se succéder. Cette évolution est un des signes de la folklorisation de la tradition indispensable à sa continuité. La journée classique évolue donc, contrairement à l'opinion savante qui la voit immuable. Ensuite sont jouées les danses du dieu de la guerre Hachiman 八幡, du dieu de la montagne Yama no kami 山の神 et de l'ouverture de la caverne rocheuse Iwato biraki 岩戸開.

La catégorisation endroit-envers et l'importance explicitement accordée aux personnages caricatures ${ }^{18}$ dits modoki 擬 font des 6 danses de l'envers des pastiches de celles de l'endroit, justifiant leurs titres similaires. Ce sont les danses des oiseaux à quatre Yonin torimai 四人鳥舞, de l'accueil du pin Matsu mukae 松迎, du troisième vieillard de l'envers Ura sanbasō 裏三番叟, du Grand Hachiman Dai Hachiman 大八幡, du dieu de la petite montagne Koyama no kami 小山の神, et enfin, de l'envers de l'ouverture de la caverne rocheuse, Ura iwato biraki 裏岩戸 開. Néanmoins, si les danses du Sanba de l'envers et du dieu de la petite montagne sont amusantes, les autres ne se distinguent guère par l'humour. Les personnages doubles de ceux de l'endroit sont vêtus de costumes semblables à ceux de leurs modèles et leur gestuelle est plus ou moins imitative. Théoriquement, les danses de l'envers se définissent aussi par un nombre de personnages doublé ; cependant, seules celles des oiseaux, de l'accueil du pin et de Sanba se conforment actuellement à ce qui semblait autrefois une règle. Les rites de l'envers sont censés être beaucoup plus efficaces car les dieux y sont plus nombreux mais surtout parce que les danses peuvent être spécifiquement destinées à soumettre les démons. Tel est le cas des danses de l'envers des courants de Noguchi, qui, selon l'érudit local Sugawara, seraient la source de la tradition de Hayachine.

Pour les danseurs, la classification en danses de l'endroit et de l'envers n'est pas toujours identique car au moins une danse, celle de l'accueil du pin, devient une danse de l'endroit au moment du nouvel an. Sasaki Naoto 佐々木直人, un danseur d'Ōtsugunai dans les années 1950, montre dans son livret de pièces que des danses appartenant aujourd'hui à la série de l'endroit faisaient alors partie de celle

18. L'idéogramme modoki 擬 peut être traduit par «pseudo »; il implique l'idée de ressemblance, de modèle. Par définition, le modoki doit imiter les gestes et la danse d'un personnage modèle. Dans la tradition de Hayachine, nous considérons trois personnages comme des modoki à des degrés divers, Sanbasō est le modoki d'Okina, Awajiwa est le modoki de Sanbasō, enfin Koyama est celui du dieu de la montagne. 
66 Cahiers d'études japonaises $n^{\circ} 21$

de l'envers et vice-vers $a^{19}$. L'exemple plus lointain du courant de Noguchi confirme ce fait. Selon les auteurs et les époques, la composition de la liste des danses cérémonielles varie. Ainsi, pour Sasaki Naoto 佐々木直人, il en existe 19 (12 de l'endroit et 7 de l'envers). Aujourd'hui, les acteurs reconnaissent uniquement les 12 danses comme cérémonielles. Ils admettent, comme l'érudition contemporaine dans son ensemble, mais en émettant des réserves, que la journée classique présente une organisation fixe : 6 danses de l'endroit omote ma $i$ 表舞 jouées de jour et 6 danses de l'envers ura mai 裏舞 habituellement exécutées de nuit. Les premières sont de l'ordre du monde visible, les secondes, moins formelles, relèvent du monde de l'invisible. Il semblerait toutefois que le nombre de danses ait été réduit de moitié puisque, d'après les archives des années Hōreki (1751-1763) :

On nommait danses de l'endroit les 12 danses de midi, et danses de l'envers les 12 danses du soir [...]. D'autre part, les 6 premières danses, du Torimai à Iwato biraki, étaient nommées « shiki mai d'introduction ». Ces 6 danses et leur ordre étaient dans tous les cas identiques, c'était pourrait-on dire une forme immuable de kagura $^{20}$.

Cette forme immuable est désignée par le terme de cérémonie fixe teishiki 定 式 par les érudits. Selon les archives citées, le rituel classique obéissait déjà au $\mathrm{XVIII}^{\mathrm{e}}$ siècle à une codification stricte du point de vue du nombre, de la succession et du contenu des danses. Bien que cette codification soit maintenant plutôt réservée aux fêtes les plus importantes, il est remarquable qu'elle ait été conservée jusqu'à nos jours. La plus grande de ces fêtes est celle de Hayachine qui se déroule au sanctuaire de Take pendant deux jours, du 31 juillet au $1^{\text {er }}$ août. Ces dates correspondent au rite de passage de l'été natsukoshi 夏越. Par ordre d'importance viennent ensuite les fêtes de clôture des danses de kagura, kagura maizome 神 楽舞ゾメ, qui marquent la fin de l'année. Elles se déroulent le 17 décembre à Take, le troisième dimanche de décembre à Ôtsugunai, puis ce sont les fêtes du

19. Voir SASAKI Naoto 佐々木直人, Ōtsugunai no yamabushi kagura 大償の山伏神楽 (Le kagura yamabushi d'Ōtsugunai), vol. 1, édition à compte d'auteur de Sasaki Yutaka, 1953.

20. Sugawara Seiichirō 菅原盛一郎, op. cit., 1979 [1969], p. 191. 
Nouvel An nommées début des danses de kagura, kagura maihajime 神楽舞初, qui se tiennent le 2 janvier à Ōtsugunai et le 3 janvier à Take. Enfin des fêtes sont offertes aux divinités tutélaires des deux sanctuaires : le 15 septembre au grand gongen d'Ōtsugunai, le 17 décembre à Seoritsu. À noter que, même pour ces fêtes spécifiques, la journée classique n'est pas toujours offerte. Elle est donc exceptionnelle. En revanche, il n'existe plus de spectacles où 12 danses sont jouées d'affilée à l'heure de midi et rejouées le soir. Aujourd'hui seulement 6 danses sont exécutées à midi, les 6 autres débutant généralement vers 17 heures. Les danses cérémonielles entrent donc dans la catégorie de l'endroit ou de l'envers selon les heures, du jour ou de la nuit, où elles sont exécutées. On remarquera que les modoki n'interviennent que lorsque le soleil décline : acteurs-dieux de l'envers, bouffons de ceux de l'endroit, ils se livrent parfois à des exhibitions sexuelles ou incarnent des démons finalement vaincus par la vertu bouddhique. La nuit est un espace-temps de licence.

Sugawara qualifie par extension les pièces de l'endroit de solaires, car elles sont jouées de jour, celle de l'envers de lunaires parce que la nuit leur est théoriquement réservée (bien qu'il ne fasse pas nuit à 17 heures en été). Ce qui revient à dire, dans la « logique »d'oppositions et de complémentarités a-un, que les premières sont associées à l'aspect masculin/un, les secondes à l'aspect féminin/a. Ceci est un exemple des correspondances religieuses qui ne font pas sens dans la réalité de la représentation mais doivent être entendues comme inhérentes au système global et clos $a$-un, fabriqué pour situer le rituel dans une perspective cosmologique et lui donner une cohérence et une pleine efficacité. La journée classique reproduirait donc l'alternance du jour et de la nuit, finalement la course des planètes, l'aspect $a$ ou un des pièces étant défini par le moment où l'on danse et non par la fonction de modèle (= endroit) ou de double (modoki= envers) des personnages. L'opposition féminin/a masculin/un n'est pas non plus pertinente, ni dans ce système de correspondances, ni dans la réalité. Dans le premier cas parce que les recueils de traditions secrètes, quels qu' ils soient (de Take, d'Ōtsugunai, de Noguchi, etc.) évoquent toujours un courant particulier unique, féminin ou masculin, et imputent cette qualification à la cérémonie, que les pièces soient de l'endroit ou de l'envers, qu'elles empruntent la manière rude (virile) aramai ou féminine douce onna mai et enfin, que le style des danseurs soit véritablement viril ou efféminé. Secundo, car cela supposerait que seules des divinités masculines 
ou féminines jouent respectivement le midi et le soir. Ce qui bien évidemment n'arrive jamais d'autant plus que certains dieux sont ambivalents...

La notion d'endroit-envers est également très variable au fil des siècles. En effet, selon la citation précédente, toutes les danses de midi, à savoir 12 pièces, sont dites de l'endroit bien que la deuxième série comporte déjà 6 danses de personnages de caractères inverses. Dans la même optique, les 12 pièces de nuit sont dites de l'envers alors que les 6 premières d'entre elles mettent en scène des personnages de l'endroit ! La notion d'endroit-envers est donc bien assujettie aux moments où l'on danse et non pas au caractère endroit ou envers des dieux qui se présentent. Au fur et à mesure qu'on les consulte, ces archives sont de plus en plus contradictoires :

Le kagura de Take commençait par la danse des oiseaux et comportait 12 danses, celle du gongen comprise, soit 12 tours ou numéros déterminés, ce qu'on appelle shiki mai $[\ldots]^{21}$.

Ici, la danse du gongen est incluse dans les 12 danses, alors que dans les autres textes, elle clôt toujours chaque série. Séparant les deux, il y avait un intermède appelé autrefois naka hairi 中入 : « c'était un court moment de repos, après les 6 shiki mai d'introduction $\gg^{22}$, précise Sugawara. Naka hairi, littéralement « entrer au centre ou à l'intérieur », pose un problème d'interprétation que nos informateurs japonais et moi-même n'avons su élucider. C'est un instant de vide provisoire, car les acteurs qui sont les dieux désertent la scène, cassant le temps mythique pour vaquer à des occupations terrestres et physiologiques. Ils ôtent leurs costumes, rentrent chez eux pour se laver, manger, se reposer éventuellement et repartent vers le sanctuaire, ce qui leur prend habituellement deux heures. Alors ils se transforment de nouveau pour entrer dans le temps où tout est permis.

Ces 6 autres danses «n'étaient certes pas organisées comme une partie de go », dit l'auteur. Elles variaient en fonction des desiderata des spectateurs ou des acteurs, comme maintenant. Il ajoute :

[...] mais leur nombre était toujours de 6 afin que l'ensemble des pièces soit de 12 : c'est ce que l'on nommait les 12 actes fixes [...], quand ces danses étaient jouées sans interruption, on disait « ne pas

21. Ibid., p. 191.

22. Ibid., p. 191. L'auteur, usant de formules impersonnelles, ne cite pas sa source. 
tirer le rideau »maku hikazu 幕引かず ou on parlait de « passage du rideau »maku tōshi 幕通.

Je n'ai jamais vu ce cas ou entendu ces expressions au sujet de la journée classique. En revanche le rideau est enlevé lors des spectacles où plusieurs troupes jouent une ou plusieurs pièces car chacune installe alors le rideau emblème de son sanctuaire.

Aujourd'hui, nous avons un total de 12 danses, réparties en deux séries s'achevant chacune systématiquement par la danse du gongen. Celle-ci est particulière en ce qu'elle est toujours identique, elle ne présente donc ni endroit, ni envers et son nom ne change pas. Par contre, le nombre de gongen peut être doublé, voire triplé, à la suite de danses de l'envers mais uniquement lors des fêtes de sanctuaire ou lorsque seule la danse du gongen est présentée. Elle est alors toujours reconnue par tous comme un rituel de kagura en soi.

Pour Honda et Sugawara, le gongen de Hayachine, à l' instar de celui de Kumano, réunirait les 12 divinités des directions et des mondes de l'endroit et de l'envers, lui-même étant situé au centre. Ils prennent pour références de la musique actuelle des archives savantes et explicites à cet égard. Les habitants reconnaissent aussi, mais implicitement, le gongen comme la divinité qui rassemble tous les pouvoirs des dieux qui se présentent, tour à tour ou simultanément, sur la scène au cours de la journée classique. Dans ce sens, le gongen est la divinité focale des rituels de Hayachine. Cependant, dans leur conception religieuse de la voie de l'ascèse, le gongen de Take incarne Seoritsu alias le bodhisattva Kannon qui gouverne l'Est (et le mont Hayachine) et non le centre.

\section{Sens du rituel : relation avec le nombre 12}

\section{Interprétations érudites}

Les études des ethnologues nationaux Orikuchi Shinobu 折口信夫 et Yanagita Kunio 柳田国男 ${ }^{23}$ montrent que le nombre 12 n'est pas fortuit. Il appa-

23. Yanagita Kunio 柳田国男, le 《 père des études folkloriques »(1875-1962) et Orikuchi Shinobu 折口信夫 (1887-1953) sont des pionniers de l'ethnologie japonaise qui ont mené des études couvrant pratiquement la totalité de l'archipel japonais sans toutefois approcher la tradition qui nous intéresse. 
raît en effet fréquemment dans l'ensemble des fêtes et des rites saisonniers japonais, évoquant le calendrier agraire. En témoigne la référence d'Orikuchi Shinobu 折 口信夫 ${ }^{24}$ à la cérémonie des lespédèzes, le mi-kamagi 御䆠木 offert respectueusement à la cour de Nara le 15 janvier (jour du petit Nouvel An) par les fonctionnaires de la cour et ceux de l'ancien régime ${ }^{25}$.

Concernant les danses cérémonielles de Hayachine, le nombre 12 est l'objet d'exégèses plus ou moins élaborées. Sugawara Seiichirō 菅原盛一郎 et

24. Voir ORIKUChi Shinobu 折口信夫, 《Kadomatsu no hanashi »門松のはなし

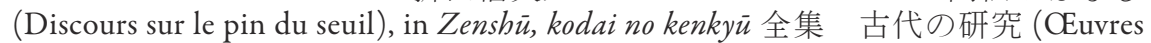
complètes, Recherches sur l'Ancien Temps), vol. 17, Tōkyō, Chūkō bunko 中公文庫, 1975 , p. 463-469.

25. C'était la règle d'apporter 12 arbres en signe de soumission à l'empereur, arbres qui devenaient pour un an les lespédèzes de la cour impériale. L'origine de cette cérémonie serait les longues branches de l'arbre nȳugi 新木, support du dieu du Nouvel An, que les montagnards rapportaient de la montagne lorsqu'ils descendaient au village à la fin de l'année pour la commémoration des morts. On y gravait 12 traits ( 13 les années bissextiles) ou bien on y inscrivait les caractères des 12 mois de l'année et on pratiquait la divination. Ce marquage n'était pas une coutume réservée aux montagnards, il était très répandu au Japon. Les montagnards pensaient que le nyūgi était l'arbre des eaux, d'où son autre nom, mizugi; selon les lieux on l'appelait aussi misogi, il était d'ailleurs indispensable pour accomplir les lustrations et aspersions d'eau chaude misogi harai et, pour Orikuchi, ce serait aussi une variété de sasaki, l'arbre sacré du shintô. Selon Gérard Martzel, Le Dieu masqué : fêtes et théâtre au Japon, Paris, Publications orientalistes de France, 1982, l'eau chaude pouvait être de l'eau de mer, les lustrations purifiant les lieux et les participants à la fête, avant la cérémonie pour les ancêtres. Orikuchi, explique que le terme $n y \bar{u}(-g i)$ vient du nom de la rivière Nyū ou Nibu qui coule à Yamato et que les dieux de cette rivière étaient les dieux des eaux. Il associe également le nyūgi à l'arbre du démon onigi qui vit dans la montagne. Par la suite serait apparu le terme de démon malfaisant jetant des sorts akkirasatsu ou akkirasetsu, et vivant au fond de la mer. Cette appellation, d'origine bouddhique, désigne un « diable » qui trompe les hommes, leur fait du mal et peut les dévorer. Il existe aussi une histoire de source bouddhiste selon laquelle ce personnage est très puissant et ses mouvements sont rapides. Il habite au pays de Rasetsu, Rasetsu koku, d'où son nom ; par ailleurs, il aide les bouddhistes à trouver la voie juste. Orikuchi propose donc diverses interprétations associées à la fois au culte de l'empereur et des ancêtres, à la commémoration des morts et enfin, au dieu du Nouvel An et au bouddhisme. Ces interprétations ne sont pas contradictoires en ce qu'elles se rapportent toutes à la revitalisation de la nature et à la restauration de l'ordre national en la personne de l'empereur. 
Honda Yasuji 本田安次 proposent ainsi leurs hypothèses. La première, ethnologique et historique, soutenue par Sugawara avant Honda, entend la journée classique comme la reproduction d'un cycle calqué sur la mythologie shintô et symbolisant la formation du pays. Elle pose en principe que la tradition des deux hameaux est issue des divertissements divins de grands sanctuaires, c'est-à-dire dérivée de kagura de Cour. La seconde hypothèse, émise par les auteurs anonymes d'archives étudiées par Sugawara, est nettement inspirée de la pensée taoïste. La troisième, formulée par Honda, reprend l'histoire du théâtre japonais et exprime l'idée que cette journée est constituée de genres panachés et recomposés. On retrouve ainsi des groupes de personnages polymorphes et ambivalents, liés à des tendances religieuses plus ou moins mêlées; elle envisage la formation de la journée classique de manière incomplète. La dernière interprétation, qui est un compromis entre les précédentes, explique la conception des indigènes et reflète «la cote » d'efficacité et par conséquent le degré de croyance qu'ils portent à chaque divinité.

\section{Première hypothèse}

Elle pose en principe que la succession des 12 danses dans un ordre donné constitue un cycle à l'image des mythes shintô et représente l'avènement des 7 générations de divinités célestes et des 5 générations de divinités terrestres. Cependant ces épisodes sont reconstruits au point d'être difficilement identifiables. Pour concrétiser leur supposition, les érudits établissent des liens entre la journée classique de Hayachine et des courants locaux qui « originairement » auraient été des kagura de Cour, donc attachés au culte de la déesse solaire Amaterasu et de l'empereur. La danse rituelle de l'ouverture de la caverne rocheuse serait le cœur de la cérémonie. Le kagura des origines (danse mythique d'Ame no Uzume) entraînant le retour du soleil, sa reproduction réinstaure l'ordre de la nature et l'ordre du pays en accroissant la vitalité et la longévité de l'empereur. Le mythe aurait donc instauré le rite. Matsumura remarque à l'inverse :

[Ce mythe] a son origine dans un rite magico-religieux effectué tous les hivers, au moment où les rayons du soleil sont les plus faibles, pour renouveler le pouvoir du soleil ; ce rite incluait des éléments du 
72 Cahiers d'études japonaises $n^{\circ} 21$

chinkon [apaisement ou fixation de l'âme] et les rires rituels ${ }^{26}$.

La cérémonie du chinkon avait une double fonction : celle de fixer puis d'apaiser l'âme, tama shizume, de l'empereur afin qu'elle n'erre plus, et la secouer, tama furi $^{27}$, pour la rappeler ou la revitaliser. Or la disparition de l'astre solaire est assimilée à sa mort ${ }^{28}$ et par extension à celle de l'empereur, admis comme son descendant. Les rites du chinkon sont donc spécialement destinés à empêcher l'âme de l'empereur de quitter son corps au solstice d'hiver.

En quête d'une forme homogène et parfaite de rituel, Honda, de même que Sugawara, privilégient ici une conception shintô qui donne des lettres de noblesse à la journée classique de Take et d'Ōtsugunai puisqu'ils la tiennent pour dérivée d'un mi-kagura. Au contraire, la tradition dont elle fait partie est revendiquée comme populaire par ses acteurs. Ce qui est le propre des kagura villageois.

Pour étayer leurs dires, les deux auteurs adoptent une démarche historicisante, diachronique, à travers laquelle ils tentent de retracer les chemins géographiques suivis par les deux courants, d'en identifier les écoles de pensée « originelles » et les agents de transmission (ascètes yamabushi pour Take, prêtres Sceau de la Loi, hōin, pour Ōtsugunai). Ils compilent et comparent des textes recopiés au fil des ans, partiels et par la suite sujets à de multiples interprétations ${ }^{29}$; ce sont essentiel-

26. Matsumura, cité dans Donald L. Philippi (dir.), Kojiki, Tōkyō, University of Tōkyō Press, 1968, p. 81.

27. Chinkon est la lecture on du terme japonais tama furi.

28. Ce mythe est la source de multiples exégèses tant au Japon qu'à l'étranger. La réclusion de la déesse solaire résulte de sa colère envers son frère ; elle est souvent interprétée comme une éclipse ou une mort symbolique. Dans de nombreux poèmes du Kojiki 古事 記, signale D. L. Philippi (op.cit., 1968, p. 81), l'expression «s'enfermer dans la porte rocheuse » signifie mourir et être enfermé dans la roche tombale. La caverne rocheuse est alors assimilée au tombeau du soleil et les rites qui s'ensuivent à des pratiques magicoreligieuses pour faire réapparaître l'astre solaire.

29. Ces copies posent maints problèmes de lisibilité et de traduction car elles furent transcrites à partir de la tradition orale, et elles furent d'abord rédigées en syllabaires hiragana et katakana. Par la suite, des sinogrammes en remplacèrent une partie, choisis en fonction de lectures dont beaucoup sont homophones. C'est pourquoi les différentes versions d'une même tradition peuvent prendre des sens divers. 
lement des recueils de tradition secrète hidensho 秘伝書 de nombreux courants régionaux de kagura. Les plus anciens recueils (difficilement) disponibles datent du XVIII ${ }^{e}$ siècle. En même temps, Honda et Sugawara se fondent sur des récits légendaires. De ces sources anonymes, ils déduisent que le kagura d'Ōtsugunai proviendrait, entre autres, du sanctuaire de Kashima ${ }^{30}$ tandis que celui de Take serait affilié à un temple de Kyōto ${ }^{31}$ ou encore rattaché au mont Haguro. D'autres ethnographes tel que Kikuchi Teruo 菊池輝男 ${ }^{32}$ pensent qu'il serait venu d'Izu, en longeant la côte Pacifique. Pour sa part, Miyake Hitoshi 宮家準 ${ }^{33}$ rapporte que :

Le desservant d'Iwate était attaché au service de l'ermitage Jikō, de la voie de l'ascèse de la tendance de la Montagne fondamentale,

30. Selon le Saibu (Zaibu) - Inbe-ryū Noguchi-ke hiden kagura honsho 斎部－忌部流 野口家秘伝神楽本書 (Véritable recueil des kagura de tradition secrète des courants Zaibu/Inbe de la Maison Noguchi), compilé par Yokogawa Gyūmon 横川儀右門 au troisième mois de l'an 6 de l'ère Kaei (l'ère Kaei couvre la période du 28/02/1848 au 27/11/1854). Il y est affirmé que le grand prêtre daigüji Norihiro, attaché au grand sanctuaire de Kashima, aurait copié la tradition relatée dans le manuscrit des « corporations Inbe Kaneharu ». Honda, qui se fonde sur le même recueil, soutient cette thèse. Il est probable que Inbe ou Imbe, provienne de imi-be, qui désigne des corporations religieuses responsables de la séclusion ou de rites d'abstinence; de même, sai-bu évoque des corporations effectuant des purifications, peut-être en rapport avec des funérailles. Le titre de daigūji est normalement seulement attribué au desservant principal du sanctuaire d'Ise. Kashima est une ville située dans le département d'Ibaraki, au nord de Tōkyō.

31. D'après nombre d'auteurs locaux, notamment KIKUCHI Teruo 菊池輝男, 《 Hayachine jinja to yamabushi kagura 》早地峰神社と山伏神楽 (Le sanctuaire de Hayachine et le Yamabushi Kagura), Matsuri 祭り (Fêtes), n 19 , Tōkyō, Société Matsuri, 1972, le moine Ennin, plus connu sous son nom posthume de Jikaku Daishi (794-864), vint à Take et y construisit le temple desservant bettō-ji nommé Myōsen, qui était en relation avec un nouveau sanctuaire situé en montagne, le shinsan-gū, d'obédience shintô. Or Jikaku Daishi est le fondateur de la branche du tendai dite « des portes de la montagne » sanmon, installée sur le mont Hiei-zan, district de Kyōto. Sous son influence et celle du moine Enchin, la composante ésotérique du Tendai se renforce, se rapprochant de la pensée shingon.

32. KikUCHI Teruo 菊池輝男, op. cit.

33. MrYAKe Hitoshi 宮家準, Nihon shükyō-shi ni okeru shugendō no ichi 日本宗教史に おける修験道の一 (Une des voies de l'ascèse dans l'histoire des religions japonaises), Tōkyō, université de Waseda, 1987, p. 7. 
dite honzan; il avait une influence extrême au sein du fief de Nanbu. Ensuite, à partir de la fin de l'époque des provinces en guerre ${ }^{34}$, l'école de pensée Haguro fut également introduite et se répandit à Ōtsugunai ${ }^{35}$.

De son côté, Sugawara ${ }^{36}$ réalise une généalogie des kagura yamabushi de la région où le courant le plus ancien (1191), celui de Minobi, aurait des liens avec la tradition de Take ${ }^{37}$. Néanmoins, il ne précise pas ses sources. En fait, les seules preuves tangibles de l'ancienneté de la tradition actuelle sont un rouleau de copies de Poèmes d'initiation secrets du kagura, Kagura hidenju-shi 神楽秘伝授詞, conservé par la famille de danseurs Sasaki d'Ōtsugunai, beaucoup plus tardif puisque daté de 1488 , et un masque signé de la même année. Ces objets datent les danses rituelles de Hayachine de manière irréfutable. Il reste par conséquent un fossé de plusieurs siècles à combler pour suivre le cheminement du rituel classique.

En bref, Honda, Sugawara et d'autres savants locaux émettent des suppositions

\section{4. $\mathrm{XVI}^{\mathrm{e}}$ siècle.}

35. La branche de la Montagne fondamentale ou du Temple principal est liée à la voie de l'ascèse de l'école Tendai, particulièrement en vigueur au temple Shōgo situé au nord-est de Kyōto. Elle s'oppose à la branche de la Vraie Montagne, ou Tōzan, rattachée à l'école Shingon et au temple Daigo sanbō qui se trouve au sud de Kyōto, sur le Mont Ōmine. La branche Honzan est supplantée au milieu du Xvi ${ }^{e}$ siècle par celle de l'école du Mont Haguro qui, à l'instar de la branche Tōzan, est affiliée au Shingon. Elle se généralise dans la région de Hayachine par l'intermédiaire des seigneurs de Nanbu, en particulier grâce au protectorat du seigneur Nanbu Toshinao qui s'étendait sur Take et soutenait son kagura, et à la popularité grandissante du Shingon au sein des familles guerrières de la région. La maison Toyotomi (famille de guerriers dont le plus illustre, Toyotomi Hideyoshi [15361598], reçut le titre de régent octroyé par la cour en 1585. En 1591, il avait presque terminé d'unifier le Japon) encourageait la tradition d'Ōtsugunai.

36. Tableau généalogique des kagura yamabushi, groupe de Hayachine. Étude de 1966 de SugaWARA Seiichirō 菅原盛一郎, op. cit., 1979 [1969], p. 7.

37. Cependant, pour MoriguCHI Tari 森口田理 (Iwate no minzoku geinō yamabushi kagura hen 岩手の民俗芸能山伏神楽編 [Recueil sur les kagura yamabushi, spectacles populaires folkloriques du département d'Iwate], Iwate, Iwate-ken kyōiku iinkai 岩手県 教育委員会, 1963), la fusion des kagura de Minobi et de Tanaka est à l'origine du kagura d'Ōtsugunai et non pas de celui de Take comme le pense Sugawara. 
pseudo-historiques changeantes au gré des textes et qui peuvent toutes être plus vraies, ou plus fausses, les unes que les autres. Elles tendent en tout cas à prouver que les courants de Take et d'Ōtsugunai ont suivi des itinéraires différents, motivant les singularités de chaque style et justifiant du même coup la relation a-un entre les deux écoles. D'autre part, elles expliquent la structure en 12 actes de la journée classique de Hayachine comme dérivant de celle des kagura de Cour pratiqués dans divers grands sanctuaires.

\section{Seconde hypothèse}

L'hypothèse précédente explique la constitution de la journée classique en 12 danses à partir d'un modèle de kagura de Cour fondé sur les mythes. Sugawara suggère d'autres origines possibles au nombre 12 , le mettant en relation avec les sources religieuses multiples du répertoire, donc également avec la mythologie :

L'origine de ces 12 shiki mai est rapportée dans un recueil de traditions transmis par leurs détenteurs et conservateurs Yanagita Yamato et Ōguni Kawauchi et leurs disciples du kagura de Tannai, et est expliquée comme suit. Il s'agit d'un document de Hōreki, An Ix (1760). 7 générations de divinités célestes, 5 de divinités terrestres, en tout 12, ensuite un an (égale) 12 mois, l'homme (a) 12 destins et le ciel 7 étoiles; sur la terre 5 voies [...].

J'ai vu danser la troupe de kagura de Tanmai ; l'organisation de sa journée classique n'est pas rapportée par l'auteur mais elle est identique à celle de Hayachine. Sugawara reprend à son compte les corrélations établies ensuite par les auteurs des archives de l'époque Hōreki :

La tête a 7 trous, le ventre 5 viscères, ce qui fait 12 destins, ce sont les éléments de l'endroit. [...] Ceci est une partie du livre de tradition donné à Oharaji Gozaemon, An IX de Hōreki, cinquième jour du deuxième mois, par le negi (desservant shintô) Tsuchinotō Minoru du sanctuaire de Tannai.

L'association avec l'anatomie témoigne d'une corrélation avec la médecine antique chinoise. Celle-ci était fondée sur une cosmologie identique à celle du 
76 Cahiers d'études japonaises $n^{\circ} 21$

Livre des Mutations, le Yi-king: alternance et complémentarité du yin et du yang, succession et transformation des cinq éléments ${ }^{38}$, etc. Les concordances établies entre les sens, les organes et les éléments cosmologiques relèvent de l'interprétation chinoise taoïste du corps humain transposée par les maîtres japonais du yin et du yang, les onmyōji 陰陽師. Il semblerait donc que les religieux japonais aient élaboré, sans toutefois l'annoncer, une théorie savante de la journée classique fondée sur la cosmologie du Tao et les rites chinois et qu'ils aient plaqué sur ce modèle des éléments de la mythologie japonaise ${ }^{39}$, ces derniers étant les seuls retenus actuellement par la mémoire locale. Les érudits omettent de préciser que la structure endroit-envers est sans aucun doute d'origine chinoise, et plus précisément issue de la structure classique des danses de la cour des Tang dont certaines devinrent plus tard les bugaku, implantées à l'origine dans la région du Kansai ${ }^{40}$. Mais poursuivons la lecture du texte de Sugawara :

Les hommes qui composent la troupe de kagura sont 12 , 7 correspondent aux 7 générations de dieux célestes et 5 aux 5 générations de dieux terrestres. Et d'ailleurs, l'éventail utilisé a 12 plis de $1,6 \mathrm{~cm}$ et $60,06 \mathrm{~cm}$ de longueur. Cela signifie 12 destinées, et, dans l'année, il y a 12 mois.

On comprend bien ici que les éléments constituant le rituel, acteurs, objets de la cérémonie, dont l'éventail n'est qu'un exemple, créent, pour Sugawara et ses sources, un système de corrélations entre le monde des dieux et le monde des

38. Bois, feu, terre, métal, eau. Voir Catherine Despeux, Taoïsme et corps humain : le Xiuzhen tu, Paris, Guy Trédaniel, 1994.

39. Souvenons-nous du grand prêtre Norihiro qui aurait recopié la tradition d'Inbe Kaneharu au début du $\mathrm{XVI}^{\mathrm{e}}$ siècle, cette tradition étant évidemment elle-même plus ancienne.

40. Le théâtre bugaku était joué à la cour chinoise des Tang et la danse de structure duelle fondée sur la conception chinoise du Yin et du Yang. Il comprenait un vaste répertoire et « était représenté sur une estrade encadrée de deux grands tambours, avec accompagnement d'un orchestre d'instruments à vent et à cordes. Élaboré à la cour de Heian, le bugaku s'est perpétué jusqu'à nos jours au palais impérial de Tōkyō, au Kasuga jinja de Nara, à l'Itsukushima jinja de Miyajima » selon l'Encyclopedia Universalis de 2000 . 
hommes qui confère sens, et par conséquent efficacité, à la cérémonie. C'est pourquoi les acteurs sont 12 et représentent les générations de dieux dont l'histoire est plus ou moins reprise tout au long des récits de chaque danse de kagura. Le discours écrit contemporain soutient cette équivalence entre le nombre d'acteurs et les générations de dieux. Dans la réalité, ce nombre est parfois supérieur ou inférieur à 12 selon les recrues. Actuellement, les deux troupes comprennent chacune 15 personnes. Selon Averbuch, le choix de 12 acteurs répond au minimum de personnes nécessaires pour que la cérémonie se déroule sans difficulté ${ }^{41}$. Elle dit que les musiciens doivent être 4 : le flûtiste, les deux joueurs de cymbales et le tambour, et il faut un récitant. Hormis ce dernier caché dans l'ombre du rideau hikage 日陰, tous sont sur scène durant l'exécution de chaque danse. Restent 7 personnes pour assurer les 12 ou 13 rôles (selon le courant) des 6 danses de l'endroit (davantage pour celles de l'envers) auxquelles s'ajoutent les rôles de 3 personnes pour la danse du gongen. Bien entendu ces rôles ne sont pas simultanés. Des danses sont exécutées en monômes, d'autres en binômes ou à 4 personnages, voire plus dans le cas des pièces de l'envers. Les rôles de musiciens et de danseurs sont interchangeables et, pour éviter la fatigue et permettre les changements de costumes, le nombre de 12 personnes serait idéal.

Il me semble pourtant que ce n'est pas pour les raisons matérielles invoquées par Averbuch que les acteurs seraient 12. Il s'agit plutôt pour les érudits d'adapter le rituel afin qu'il forme un ensemble clos cohérent bien que provenant d'éléments religieux remarquablement disparates. Les chiffres sont particulièrement intéressants à cet égard, le 12 en particulier, puisqu'il est manipulable dans tous les cas (dans la pensée chinoise, le culte shintô, la voie de l'ascèse, etc.), et dans tous les sens : ses multiples et diviseurs sont également très utiles pour relier ces divers concepts religieux entre eux.

Le texte précédent rattache les éventails au nombre 12 mais actuellement ils ne comportent que 10 baguettes. En outre, il n'y a pas que le nombre des acteurs qui soit abusivement identifié par les auteurs japonais à celui des générations divines. L'exemple est frappant en musique. Ainsi, Sugawara mentionne que :

41. Irit Averbuch, Yamabushi Kagura: A Study of a Traditional Ritual Dance in Contemporary Japan, Michigan, U.M.I, 1989. 
Les rythmes à 7 temps correspondent aux 7 générations de dieux célestes, les rythmes à 5 temps correspondent aux 5 générations de dieux terrestres ${ }^{42}$.

Les érudits contemporains mettent également des « rythmes à 5 et 7 temps », go byōshi, nana byōshi, en relation avec les 5 générations de divinités terrestres et les 7 de divinités célestes et, entre autres, les qualités a-un des deux écoles. Pour eux, ces temps singulariseraient les musiques respectives de Take et d'Ōtsugunai. Pourtant le rythme est binaire et, à l'écoute, les différences perceptibles le sont uniquement dans le style. Les villageois, acteurs ou non, répètent inlassablement la même chose sans rien y comprendre. La porosité de la théorie savante est ici évidente mais personne n'est en mesure d'apporter des éléments pertinents du

42. Sugawara Seiichirō 菅原盛一郎, op. cit., 1979 [1969], p. 183. Cette citation mentionne ensuite que : « les 3 rythmes correspondent aux 3 dieux de la création ». Leur identité demeure très floue, ils sont simplement nommés les 3 divinités, mi hashira no uzu no miko no kami. Cette précision souligne cependant qu'il existait en outre un groupe de 3 mesures mis en parallèle avec les 3 dieux de la création. Honda Yasuji 本田安次 évoque aussi ces 3, 5 et 7 rythmes dans Yamabushi kagura, bangaku 山伏神楽 番楽 (Kagura yamabushi, Bangaku), Tōkyō, Iba shoten 井場書店, 1971 [1942], p. 4 : « les kagura et les bangaku ont 3 mesures : 3, 5, 7 rythmes; ensuite les 3 et 5 ont peu à peu disparu, donnant d'autres formes... ». L'existence de 3 rythmes n'est pas surprenante car ce groupe de chiffres, 3-5-7, est extrêmement courant dans les rituels japonais, notamment dans les kagura. On remarque que ces rythmes étant ici identifiés aux générations des dieux, leur somme n'est plus 12, chiffre supposé déterminer les éléments de la journée classique de kagura, mais 15. Signalons par ailleurs que ce nombre ne fait l'objet d'aucune théorie savante. Il est possible que ces 3 dieux soient représentés par les 3 danses de vieillards, Okina, Sanbasō et Matsu mukae de la tradition d'Ōtsugunai-Noguchi-Zenmyō. À Take, la tradition tient également compte de ces 3 dieux de la création. D'autres traditions les assimilent au nyorai, le bouddha Tathāgata, sous ses 3 aspects de la Loi, de la Rétribution et de l'Adaptation. Voir MiYake Hitoshi 宮家準, Shugendō girei no kenkyū 修験道儀 礼の研究 (Étude sur les rites de la voie de l'ascèse), Tōkyō, Shunjūsha 春秋社, 1985, p. 198-212. Dans Kojiki il est question des 5 générations de dieux célestes précédant les 7 générations de dieux terrestres. Amaterasu, la déesse solaire, y fait partie de 3 dieux engendrés par Izanagi mais, entre les versions de ce texte (Donald L. Philip pi [dir.], op. cit., 1968, p. 47-54 ; p. 75) et du Nihongi (William George Aston, Nihongi, Rutland - Tōkyō, Charles E. Tuttle, 1972, p. 1-25), règne une grande confusion au sujet de ces mythes de création. 
point de vue musical ! Cette théorie que j'ai étudiée de très près est visiblement inspirée par le système musical chinois, notamment par un rituel des Zhou remarquable par sa complexité. Cette sophistication est incompatible avec la musique, rudimentaire, des kagura de Hayachine!

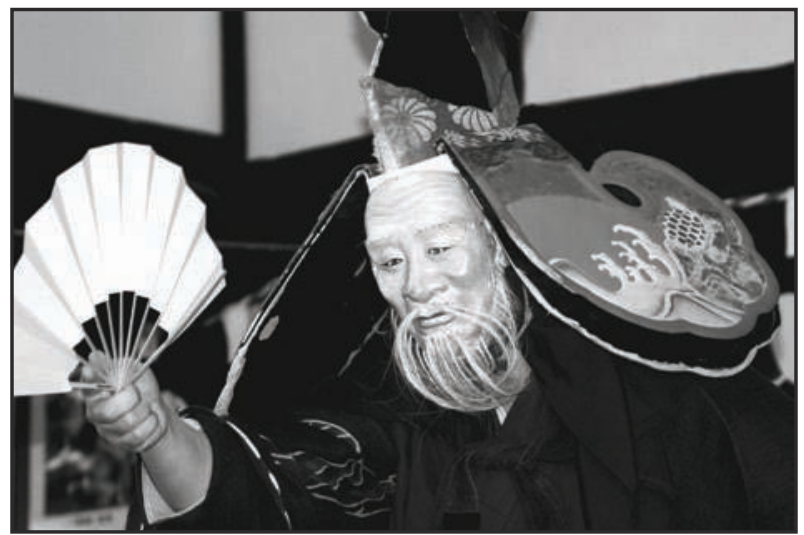

FIGURE 2.

Ōtsugunai, 2 janvier 2007, danse du vieillard nommé « Accueil du pin », Matsu mukae. Danse particulièrement offerte au Nouvel An, pour apporter longévité et bonheur aux hommes.

Source : cliché de Catherine Delpuech

Parmi les autres éléments relatifs au nombre au 12, le dieu de la montagne, dont la place est essentielle dans les croyances locales et le rituel des kagura, ne doit pas être oublié parce qu'il est parfois appelé « Dieu Douze ». Il est fêté, à Ōhasama et dans nos deux villages, le 12 février et le 12 décembre, soit le 12/12. Or, en Chine et au Japon, on pense couramment que les chiffres identiques redoublés sont fastes. À propos de la fête (hivernale) du dieu de la montagne en général, Honda mentionne que ce jour-là, les hommes doivent se rassembler et fabriquer du saké qui est offert au dieu avec 12 boulettes de farine de riz crues ${ }^{43}$. Le tout est posé sur l'autel des dieux. Une cérémonie identique a lieu au début du printemps.

43. Honda Yasuji 本田安次, op. cit., 1971 [1942], p. 435. 
80 Cahiers d'études japonaises $n^{\circ} 21$

Ces 12 boulettes représentent les 12 mois de l'année, auxquels le dieu de la montagne donnerait naissance et qui sont considérés comme ses enfants. On revient donc à la conception calendaire.

En résumé, les religieux, auteurs des recueils de traditions secrètes, rattachent, sans le dire, le nombre 12 des danses cérémonielles à une origine tao (voire antérieure). À celle-ci, ils superposent la cosmologie shintô, les croyances dérivées de la voie de l'ascèse (à travers le culte du dieu de la montagne) et les coutumes montagnardes. Ils adaptent donc systématiquement numérologie, courants religieux et danses cérémonielles du kagura. Les érudits qui s'appuient sur leurs écrits (Honda, Sugawara, etc.) poursuivent dans ce sens.

\section{Troisième hypothèse}

Honda avance une autre hypothèse qui contredit la première en ce qu'elle infirme l'idée de l'homogénéité shintô de la journée classique. Il suggère que la structuration en 12 danses de ce rituel de Hayachine provient de l'histoire du théâtre japonais, se rapprochant dès lors de l'une des interprétations locales ${ }^{44}$. Le nombre de danses aurait d'abord été de trois ${ }^{45}$, reproduisant l'ancienne partition « en 3 actes cérémoniels » dits shiki sanban des spectacles de divertissements de rizières dengaku et de singeries inspiratrices du nô ${ }^{46}$, les sarugaku no nō ; partition qui existe par ailleurs dans les kagura de Cour. Trois vieillards, Okina, Sanba et Manzai (alias Banzei) se présentent successivement sur les scènes de kagura. Au fil des ans, le dernier personnage adopte diverses identités dans les représentations de nô. Cependant Honda pense que les 3 actes à l'origine de la journée classique de Hayachine étaient différents de ceux des kagura de Cour parce qu'il entend ici le

\section{Ibid., p. 60-61.}

45. Honda ne donne pas de dates précises, il mentionne seulement « avant la restauration du nô », ce qui suppose le XIII ${ }^{\mathrm{e}}$ siècle.

46. Les singeries ou sarugaku furent à l'origine des grands genres du théâtre japonais, notamment du nô. Les divertissements de rizières dengaku étaient parfois considérés comme des kagura s'adressant principalement aux dieux agraires et locaux. 
mot shiki, non pas au sens de « cérémonie » 式, mais de «plateau » ${ }^{\text {盆 }}{ }^{47}$. Selon lui, ce nom proviendrait de la danse du plateau shiki mai qu'un acteur exécutait en portant un plateau où reposait un masque qu'il mettait pour la pièce suivante ${ }^{48}$. Nous n'avons pas d'autres références à ce sujet. Pour Honda, le rite en 3 actes de Hayachine était donc constitué d'abord de cette danse, jouée visage découvert, qui préparait la venue des dieux, puis des danses d'Okina et de Sanbasō. Ce qui suggère que le masque du plateau était celui d'Okina mais, curieusement, Honda ne le confirme pas.

Si cette hypothèse est juste, on avait d'abord une danse où l'acteur présentait le masque et l'utilisait comme torimono, actualisant la présence du dieu. La danse autour du masque du vieillard faisait ainsi valoir, dès le début du rituel, le pouvoir d'Okina qui est d'accroître la longévité. On peut donc assimiler cette danse du plateau à celle d'Okina lui-même. Dans la deuxième danse, Okina était totalement incarné par l'acteur. Ensuite intervenait son double, sa caricature modoki ou la représentation d'un Okina jeune et fougueux, le dénommé Sanbasō.

L'usage du plateau au cours d'une danse actuelle du nom d'O-shiki (o est une particule honorifique) suggère l'analogie entre les deux danses. Néanmoins l'idéogramme shiki signifie là « cérémonie » et non « plateau », ce qui va non seulement à l'encontre de l'interprétation de Honda mais de celle de l'acteur Abe Teruo 阿部輝雄, d'Ōtsugunai, pour qui (et il n'est pas le seul) cette danse n'a rien de rituel. En effet, il affirme que :

C'est une attraction qui est donnée à n'importe quelle occasion, uniquement pour distraire le public. Elle est très courte (quinze minutes), toujours jouée seule, un acteur y exécute des figures acrobatiques en tenant un plateau, mais sans masque.

47. Il existe moult graphies du mot shiki mais la plus courante dans les textes sur la tradition signifie 《cérémonie », « rite », « système ». La lecture habituelle de « plateau » est bon mais Honda précise la lecture shiki du caractère. Le bon fait référence à la fête des morts, Bon matsuri, et par conséquent aux danses données à cette occasion. La danse du plateau, shiki mai, permettait peut-être de faciliter le passage de l'âme du défunt vers le monde des ancêtres symbolisé par le masque d'Okina.

48. Cette danse existe encore sur la côte nord-ouest, répertoriée parmi les divertissements alternés bangaku. 


\section{CIPANGO}

82 Cahiers d'études japonaises nº 21

Par ailleurs, Honda compare l'ancienne danse du plateau à la danse de l'œuf Keiran mai (tradition Noguchi-Zenmyō ${ }^{49}$ ) aussi appelée Tori mai (tradition actuelle) en insistant sur leurs fonctions identiques de purification et d'appel des dieux sans motiver ces qualités quant à la première. Cette similarité, si elle existe, ne suffit pas pour justifier que la danse des oiseaux ait remplacé celle du plateau. En effet, en admettant que la danse ancienne du plateau soit la même que celle d'O-shiki, le nombre d'acteurs et la chorégraphie sont différents de ceux de la danse des oiseaux. En outre, ces deux danses, Shiki mai et $O$-shiki mai, n'introduisent pas une série et la seconde est profane pour les acteurs. L'hypothèse de Honda selon laquelle l'organisation actuelle du rite provient de 3 actes, danse du plateau comprise, paraît donc peu crédible. Enfin, l'auteur ne suggère pas la manière dont le rituel de 12 danses se serait constitué par la suite. Ces 3 actes existaient-ils déjà en double (endroit et envers) ? Comment les 3 danses suivantes (Hachiman, dieu de la montagne et ouverture de la caverne) se seraient-elles greffées sur les 3 premières dans les deux séries ? Ou bien les 6 danses de l'endroit formaient-elles déjà un ensemble et servirent-elles de modèles aux pièces de l'envers? Il est tout simplement impossible, et vain par ailleurs, de savoir comment le rituel s'est constitué dans le temps. Honda s'y attache parce qu'il suit une démarche historique, à la recherche de traces de survivance du passé dans le présent, comme la majorité des folkloristes japonais.

\section{L'interprétation villageoise}

Interrogés, les villageois, dont les acteurs, montrent peu d'empressement à expliquer la genèse des 12 danses de la journée classique. L'important à leurs yeux est avant tout le plaisir de participer aux représentations, de perpétuer la tradition transmise par leurs ancêtres, de danser et de se retrouver entre amis. Nonobstant, ils associent la constitution de la journée classique en 12 actes aux mois de l'année, aux générations des dieux et à la formation du pays expliquée dans le Kojiki (pour ceux qui l'ont lu). Ainsi le monde des hommes et de la nature refléterait explicitement le temps mythologique et le monde des dieux. Le dieu de la montagne, alias Dieu Douze, relie ces deux mondes.

49. Citée par Sugawara Seiichirō 菅原盛一郎, op. cit., 1979 [1969], p. 176-192; Honda Yasuji 本田安次, op. cit., 1971 [1942], p. 564 et suivantes. 
La plupart ignore les textes et courants de kagura auxquels les érudits se réfèrent. Ils admettent bien évidemment que des pièces du rituel rejouent des épisodes mythologiques : ils voient Izanagi et Izanami dans le couple de la danse des oiseaux et identifient le mythe de l'ouverture de la caverne rocheuse. Sanba est bien la larve-sangsue Hiruko et Awajima (Sanba de l'envers) l'île du grain de millet. Cependant, ces connaissances, très limitées, sont sans aucun doute issues des discussions avec les danseurs, eux-mêmes formés oralement par leurs maîtres, instruits par les livrets en circulation et, pour certains, par leur curiosité personnelle, car ce ne sont pas les textes étudiés à l'école qui tissent des analogies entre ces dieux mythiques et ceux qui dansent leurs kagura ${ }^{50}$. Mes entretiens avec les villageois, y compris les danseurs, montrent qu'en majorité ils connaissent mal la mythologie. Cela s'explique par le fait qu'ils ne valorisent « les dieux nationaux » que sous leur aspect visible dans la danse et l'efficace de son exécution : ainsi la danse de Sanba favorise les bonnes récoltes mais Hiruko ne leur apporte rien, Okina prolonge leurs jours mais ils ne connaissent pas le dieu Ame no Koyane qui le représente dans les mythes. Cette ignorance est due aussi à l'hétérogénéité des pièces, tant au niveau des textes que de la gestuelle. Chacune est en effet un amalgame de bribes de mythes ponctuées de références souvent obscures au bouddhisme, de rites magico-religieux, de brassages de courants de kagura et d'autres genres théâtraux, dont il est difficile de démêler les influences respectives.

Cette confusion reflète la conception du divin au Japon, soulignant qu'il existe un fond religieux commun d'où ressort l'idée générale qu'une divinité n'a pas une existence, une identité, ni même un genre fixe. Pour ceux que j'appelle les gens du kagura, c'est-à-dire les villageois et acteurs-villageois, ces deux critères comptent peu finalement : les dieux sont interchangeables, ce sont surtout leurs bienfaits qui sont appréciés. Okina est un exemple type de ces mélanges, il relie tout, son efficacité rituelle est toujours très simple, il lui suffit de taper des pieds pour que la nature se réveille et que la terre soit fertile. La perplexité des villageois quant à la nature des personnages joués dans la journée classique (et dans les rituels cou-

50. Sasaki Takashi, Abe Teruo d'Ōtsugunai, ou Kaneto l'intellectuel de la troupe de Take, se sont aussi penchés sur les thèses contemporaines et ils en parlent bien qu'ils nient leur intérêt pour tout ce qui est écrit. 
rants) laisse à penser que les règnes sont perméables : certains personnages a priori divins sont aussi animaux ou humains, voire les deux ou les trois à la fois. Les masques et les chants divins sont d'ailleurs révélateurs de ces multiples facettes. Les vieillards sont des ancêtres mais parmi eux, Sanbasō présente toutes les qualités de la jeunesse et l'agilité du singe. Dans son ensemble, le groupe des vieillards est une « bande de jeunes », de corps et d'esprit. Les ancêtres réels qui, des années après leur mort, deviennent normalement dieux, ne sont pas vraiment considérés comme tels dans le rituel par les acteurs.

La danse de l'ouverture de la caverne autour de laquelle s'organise le rituel selon les conceptions savantes joue un rôle mineur dans l'esprit local, sans doute parce que les dieux en présence « ne sont pas des dieux d'ici », disent les villageois : les dieux locaux sont bien sûr les meilleurs. Les dieux nationaux n'ont aucun effet sur leur vie au contraire du dieu de la montagne et du gongen qui exercent une fascination sur tout le monde. Véritables divinités des gens de Hayachine, c'est à elles qu'ils adressent leurs prières, dansées ou non, car ils éviteront un malheur ou y gagneront quelque chose, croient-ils. En revanche, ils ne font pas de liens entre la cérémonie et les 12 destins qui seraient affectés à l'homme bien que l'exécution de chaque danse soit susceptible d'influencer leur avenir. Quant aux 7 étoiles et aux 5 voies évoquées dans les archives et probablement dérivées de la vison chinoise organiciste du monde, ils n'en ont jamais entendu parler.

Ils ne doutent pas que la danse du gongen ait précédé la formation de la journée classique. Certains pensent même que les légendes détiennent une parcelle de vérité et font donc remonter son « apparition » au début du IX ${ }^{\mathrm{e}}$ siècle (807). La danse du dieu de la montagne Yama no kami, et celle de son double Koyama sont aussi pour eux très anciennes mais personne n'avance de dates, ce qui est d'ailleurs impossible. Liées également aux rites de la voie de l'ascèse, ce sont des danses de prières d'exorcisme dont la première est reconnue comme très efficace.

Ils identifient donc des groupes de personnages : les divinités locales (dieux de la montagne et gongen), les vieillards et les dieux du shintô. Il est d'ailleurs intéressant de souligner qu'à Take, les danseurs ignorent que Seoritsu est la «vive manifestation » de la déesse solaire Amaterasu, ce qui confirme leur peu d'intérêt pour cette dernière. À Ōtsugunai, ils savent cependant que leur gongen incarne les trois dieux du sanctuaire, par conséquent des kami de la mythologie shintô. Malgré cela, les gongen sont toujours pour les uns et les autres avant tout des divinités locales 
probablement (à mon avis) parce que, hormis leur rôle de divinités tutélaires, ce sont des dieux de lignée qui sous leur forme fantastique de serpent-dragon représentent les ancêtres. C'est d'ailleurs pour cette raison que leur territoire ne peut être aliéné par d'autres gongen et que la forme de leur être ne peut être cédée même provisoirement : en effet, seule la danse des gongen de Hayachine est autorisée dans la montagne et les têtes de shishi de ces kagura ne peuvent être empruntées.

Les danseurs des deux écoles rejoignent l'hypothèse de Honda quant à l'idée que le groupe des vieillards viendrait du shiki sanban habituel du nô, à ceci près qu'ils n'intègrent pas la danse du plateau à ce processus. La facture des masques d'Okina et des personnages de l'accueil du pin est d'ailleurs très proche entre les deux genres théâtraux. Il est cependant probable que ces personnages qui miment des pratiques agraires (semailles) ou des coutumes (l'accueil du pin au nouvel an) aient « vécu d dans les campagnes bien avant d'être interprétés formellement sur les scènes de nô. Ensuite, des pièces appartenant au répertoire national et tirées de la mythologie (celle des oiseaux, de Hachiman, de l'ouverture de la caverne, ainsi que leurs contreparties de l'envers et les danses de démons) auraient été ajoutées. Personne ne sait ni ne se préoccupe de savoir quand ou comment elles se sont agglutinées avec les précédentes pour former la journée classique. Certains pensent néanmoins qu'elles auraient été importées de temples de Kyōto, transmises par les yamabushi. D'autres font remarquer, justement, que le rideau de scène d'Ōtsugunai est décoré de deux armoiries, un chrysanthème sur la gauche (blason mon de la maison impériale) et, sur la droite, trois feuilles de paulownia entourées de 5 fleurs et surmontées de 7 au centre (blason de la maison Toyotomi) ${ }^{51}$, ce qui confirmerait des liens avec des kagura de Cour, qui restent cependant inexpliqués $^{52}$. Ce qui est sûr est que la tradition était, et demeure, transmise par une lignée de bettō de la famille Sasaki. D'autre part, les liens avec un des courants Noguchi sont avérés dans la mesure où une tombe porte ce nom dans le cimetière d'Ōtsugunai.

Deux grues de profil aux ailes déployées ornent le rideau de scène de Take.

51. Cette formule 3-5-7 est auspicieuse et existe partout au Japon, ainsi les enfants fêtent les âges de 3, 5 et 7 ans en se rendant au sanctuaire pour être bénis.

52. ICHINOKURA Shun.ichi一/倉俊一, op. cit., 1995, p. 36-39. 
86 Cahiers d'études japonaises $n^{\circ} 21$

Elles se font face, l'une ayant le bec entrouvert, l'autre le bec fermé, conformes à l'opposition ouvert/fermé a-un, qui caractérise les relations entre les deux écoles. La grue est connue pour être un symbole de fidélité mais l'origine de ce motif est ignorée. Elle pourrait avoir un rapport avec le blason mon des seigneurs Nanbu qui protégeaient la bourgade et encourageaient son kagura. Ces danses « nationales » auraient par conséquent pu venir du pays de Kaii où les Nanbu avaient aussi un fief, jusqu'en pays d'Ōu et de là, passer à Hayachine.

Les spéculations quant à la constitution en 12 danses de la journée classique sont surtout le fait de l'érudition. Le sens du rituel pour les villageois peut être résumé comme suit. La danse des oiseaux est essentielle par son rôle de purification qui permet la descente des dieux. Elle favorise, d'autre part, l'entente conjugale et la fidélité des couples. La danse d'Okina est surtout appréciée des plus vieux, elle leur accorde la longévité ainsi qu'à leurs descendants. Celle de Sanba assure la germination au printemps et plaît à tous par sa fantaisie et les prouesses de l'acteur. La danse de Hachiman, moins spectaculaire, suscite des réactions moins vives et moins intéressées dues à l'absence de masque. Pour la tradition savante, elle protégerait le territoire des influences néfastes, mais elle n'a pas de valeur particulière pour les habitants. Le dieu de la montagne est toujours très attendu, des enfants auxquels il jette des friandises, mais aussi des adultes qui lui prêtent de nombreux pouvoirs. Il promet, entre autres, des accouchements faciles et de bonnes récoltes sous sa forme de dieu des rizières. La danse de la caverne est appréciée esthétiquement car elle met en jeu beaucoup de personnages masqués et des costumes variés. Toutefois, elle ne suscite pas de croyances particulières, d'autant moins l'idée que son exécution inaugurerait la renaissance du monde. Certaines danses de l'envers amusent beaucoup la galerie, celle du dieu de la petite montagne rencontrant le plus de succès. Son exécution est aussi, comme celle de son modèle, gage de fertilité et de fécondité.

À chaque fois, le rituel recommence par la danse des oiseaux identifiée par les religieux d'autrefois au chaos et s'achève par le retour au $\cos \operatorname{mos}{ }^{53}$. Enfin, le gongen apparaît, divinité de l'eau maîtrisant le feu, serpent dragon siffleur symbolisant le cycle éternel des renaissances, à l'instar du rite du passage à travers la

53. Sur la notion de chaos et de cosmos, consulter Herbert E. Plutschow, "Chaos and Cosmos" Rituel in Early and Medieval Japanese Literature, Leiden, E. J. Brill, 1990. 
matrice tainai kuguri 胎内くぐり effectué par les villageois au franchissement de l'été. En définitive, il apparaît que pour les acteurs au sens large l'ordre des danses suit une logique progressive quant à leurs vertus axées sur l'idée fondamentale de perpétuation des espèces et de renouvellement de la vie. La journée classique prend sens en ce qu'elle concentre dans une unique cérémonie les pouvoirs qu'ils attribuent à chaque danse et leur assure ainsi une protection globale contre les aléas de la vie.

Malgré cela, elle n'est pas pour moi représentative de la tradition. Preuve en est son caractère exceptionnel déjà mentionné. Elle est d'autre part offerte deux ou trois fois par an lorsque viennent des étudiants de Tōkyō (à Ōtsugunai) ou de petits groupes de touristes organisés, prenant alors une valeur quasiment pédagogique. La centaine de spectacles auxquels j'ai assisté me permet aussi d'affirmer qu'elle n'est pas la règle qui fonde les rituels de Hayachine. Son exécution requiert cinq heures, ce qui explique certainement aussi sa rareté. Les acteurs, « pris dans le tourbillon de la vie », jonglent avec le temps, et les spectateurs supportent moins bien qu'autrefois les longs spectacles. Les cérémonies ont évolué dans leur forme, ordre et contenu, mêlant danses cérémonielles et non cérémonielles du répertoire de kagura, pièces comiques kyōgen et divertissements alternés bangaku ${ }^{54}$. De plus, à l'exception des 6 journées spéciales de kagura, il y a rarement un rituel préliminaire de purification au sanctuaire, d'autant moins quand les danses se passent en dehors des villages. Les spectacles comprennent de une à plusieurs danses, sachant que lorsqu' il n'y en a qu'une, il s'agit de celle du gongen. Quand le rituel se compose de plusieurs danses, le Torimai tient toujours lieu de rite de purification.

Le nombre des danses dépend des contingences, en priorité des danseurs disponibles, ensuite de la nature et du lieu du spectacle. Lorsqu'il est joué par une seule troupe dans la salle des fêtes ou le pavillon de kagura, il dure en moyenne deux heures, soit le temps d'interpréter quatre à six danses. Dans ce cas, c'est la troupe qui, en accord avec son chef, compose le programme. Pour la grande fête de Hayachine, le temps est limité par les instances religieuses car plusieurs groupes interviennent. À l'extérieur des villages, ce sont les organisateurs qui l'imposent.

54. Les bangaku, surtout visibles sur la côte ouest du Japon, ont une origine commune avec les kagura. 
Le choix des danses peut être laissé à des particuliers qui mandent les troupes, autrement les acteurs se consultent. Il arrive qu'il soit modifié juste avant le spectacle si une requête est faite par le public. L'exécution spéciale de la journée classique pour les touristes montre bien quant à elle que c'est finalement ce rituel qui se constitue en attraction. En réalité, n'importe quelle pièce peut être jouée mais le gongen doit toujours apparaître et danser. Enfin, certaines danses sont privilégiées en fonction des saisons, par exemple Okina et l'accueil du pin au nouvel an, Sanba au printemps, Amaterasu aux cinq céréales en été, l'ouverture de la caverne rocheuse en hiver. En cela, on peut dire que les rituels courants conservent un contenu principalement assujetti au calendrier agraire et révélateur des croyances inhérentes. J'ai noté un raccourcissement plus ou moins important des textes par rapport aux versions plus anciennes, et donc une perte de sens. La gestuelle des danses des rituels courants s'est également modifiée de même que celle des danses de la journée classique. Dans l'ensemble, l'évolution des rituels est due en grande partie au manque de temps et de disponibilité des danseurs et à l'influence d'un public élargi. Un modèle plus proche du folklore lui est donc offert, signe d'adaptation aux conditions de vie actuelles qui est nécessaire à la continuation de la tradition de Hayachine. Cette tendance à la folklorisation et à la théâtralisation (les troupes jouent en effet souvent dans des théâtres) n'empêchent pas les danseurs de considérer le kagura comme un art sacré. Les restrictions toujours en vigueur quant à l'apprentissage et à la pratique sont révélatrices à cet égard.

\section{Conclusion}

J'ai parlé d'interprétation « érudite » pour définir principalement la pensée véhiculée par les textes et les ethnologues japonais, opposant cette tradition écrite à l'opinion locale. Bien évidemment, cela ne signifie pas que cette dernière est dénuée d'érudition mais qu'elle fonctionne sur un autre plan, celui de l'expérience corporelle. Elle met en jeu l'immédiateté des actes que les danseurs, et leurs communautés respectives, opèrent dans le sens de la vie de la tradition. Elle donne en outre, un sens à leur vie sociale et personnelle parce que le kagura est une passion et qu'il les valorise.

Ces deux conceptions représentent a priori deux mondes séparés qui s'ignorent plus ou moins. Cependant, le filtre est imparfait dans un sens. En effet, 
les érudits ne consultent aucunement les acteurs, ce qui amène les questions très actuelles de l'interprétation en ethnologie, de l'implication de l'ethnologue, et des conséquences sur le milieu étudié. Dans la tradition de Hayachine, celles-ci se manifestent par l'intégration totale de certaines notions savantes, depuis fort longtemps, à la pensée autochtone. Il en est ainsi concernant l'opposition et la complémentarité a-un des courants de Take et d'Ōtsugunai. Cette entité, cultivée quasiment à tous les niveaux, s'exprime à travers les moyens que les yamabushi ou assimilés ont instaurés pour implanter la voie de l'ascèse à Hayachine. Ces moyens suivent un schéma classique existant en des lieux similaires, c'est-à-dire montagneux, investis par le shugendō. Ainsi, les anachorètes inventent des anecdotes, bâtissent des légendes (ou utilisent celles qui ont déjà servi) où, sous l'apparence d'hommes ordinaires, ils se mettent en scène et sont illuminés par des apparitions divines. De hameaux en hameaux, et de maisons en maisons, ils font danser les divinités, de l'avis des autochtones d'abord le gongen et le dieu de la montagne. Elles deviennent alors indissociables du paysage quotidien des villageois qui leur consacrent un véritable culte. Ensuite, ils structurent l'espace, bâtissant des temples et des sanctuaires reliés les uns aux autres, auxquels des $z a$ exécutant les kagura sont rattachés.

Il y a de quoi se perdre dans les méandres des reconstitutions érudites. Les auteurs insistent sur les origines des danses de kagura, ce dont les acteurs se soucient peu, ainsi que leurs constructions historiques. Elles motivent le modèle a-un, répandu non seulement dans la région mais, on l'imagine, partout au Japon, cependant la disposition géographique de nos deux villages, opposant plaine et montagne, s'y prêtait particulièrement. On s'aperçoit que ce modèle est fondamental parce que tout le monde en parle, c'est la première chose que les touristes nouveaux venus dans la région apprennent. Il explique donc encore aujourd'hui la concurrence entre les deux courants et, par conséquent, il reste l'un des facteurs essentiels du maintien et de la vigueur de la tradition.

L'analyse des 12 danses de la journée classique montre que le rituel, glosé par les érudits, met en relation temps cosmogonique et temps cyclique. Il répond à la volonté d'ajuster les danses aux étapes de la création du pays par les kami et au cycle agraire, pour être en adéquation avec une vision du monde déterminée dans ses grandes lignes par le culte shintô. En effet, pour les lettrés, les divinités qui dansent incarnent une idéologie de protection de l'empereur (= État), en revanche 
pour les habitants, il n'en va pas de même. Ils savent que certaines de ces danses rejouent des épisodes mythologiques mais peu importe l'identité des dieux qui se présentent, ce sont les pouvoirs qu' ils accordent à l'exécution de leurs danses dans l'observation des codifications déterminées par la tradition qui priment. En fait, ils pensent que si les rites ne sont pas accomplis dans ces formes, toutes sortes de calamités s'abattront sur la communauté. L'idée de consubstantialité de la pratique du kagura avec la nature reste prégnante dans l'interprétation locale, l'environnement étant conçu comme un facteur favorisant les vocations. En extrapolant à peine, le corps de l'acteur est encore, dans la pensée actuelle, assimilé à celui des dieux et, par extension, à la Nature.

Si l'ensemble de la chorégraphie est toujours entendu par les érudits comme une succession de prières en mouvement, il ne fait pas en tant que tel l'objet des croyances, c'est sa réalisation seule qui conjure le mauvais sort. L'idée principale est donc intimement liée au corps agissant, offert aux dieux dans un jeu quasiment séducteur, puisqu'il s'agit de les divertir par des danses accompagnées de poèmes de louanges et de musique pour en obtenir une protection préventive contre des malheurs éventuels. Le but de la réalisation et de la répétition, voulue comme identique, des rituels (classique ou non), touche aussi au problème de la conservation de la tradition. Le point de vue des acteurs et de leurs spectateurs est par conséquent beaucoup plus concret que celui des lettrés. Dans l'esprit des premiers, rite et spectacle se confondent. Ils ont effectivement conscience d'accomplir des gestes rituels et ils le font le mieux possible, cependant ils souscrivent à leur désir de plaire à un public qui n'est pas toujours le leur et organisent des spectacles en conséquence, préférant au rituel classique des programmes plus amusants ou, au contraire, l'utilisation de ce même rituel dans une optique quasiment pédagogique. C'est aussi une manière de transmettre le kagura. Par leur passion qui s'exprime à travers leur dynamisme, leur savoir a résisté à l'érosion du temps. L'ethnologue Honda a participé activement à cette lutte. Néanmoins, les érudits dans leur ensemble, lui inclus, donnent à lire des textes insistant sur l'idée d'un modèle immuable, clos et parfait, lié aux mythes et à l'histoire, et que rien ne saurait remettre en question. Cette vision figée ne correspond pas à la réalité de la tradition de Hayachine. Les danseurs ont bien compris la nécessité d'être en accord avec leur temps pour qu' elle perdure. 


\section{Bibliographie}

Aston William George, Nihongi, Rutland - Tōkyō, Charles E. Tuttle, 1972.

Averbuch Irit, Yamabushi Kagura: A Study of a Traditional Ritual Dance in Contemporary Japan, Michigan, U.M.I, 1989.

Despeux Catherine, Taoïsme et corps humain: le Xiuzhen tu, Paris, Guy Trédaniel, 1994.

HondA Yasuji 本田安次, Yamabushi kagura, bangaku 山伏神楽 番楽 (Kagura yamabushi, Bangaku), Tōkyō, Iba shoten 井場書店, 1971 [1942].

HoNDA Yasuji 本田安次, Nihon no minzoku geinō 日本の民俗芸能 (Spectacles populaires folkloriques du Japon), vol. 5, Tōkyō, Mokujisha 木耳社, 1966.

Honda Yasuji 本田安次, Minzoku geinō, kagura 民俗芸能 神楽 (Spectacles populaires folkloriques, kagura), Tōkyō, Bunkachō 文化庁, 1970.

ICHINOKURA Shun.ichi一 一八倉俊一, Hayachine kagura 早池峰神楽 (Les kagura de Hayachine), Morioka, Ōhasama-machi kankō kyōkai 大迫町観光 協会, 1995 .

KIKUCHI Teruo 菊池輝男, 《Hayachine jinja to yamabushi kagura 》早地峰 神社と山伏神楽 (Le sanctuaire de Hayachine et le Yamabushi Kagura), Matsuri 祭り (Fêtes), nº 19, Tōkyō, Société Matsuri, 1972.

Martzel Gérard, Le Dieu masqué : fêtes et théatre au Japon, Paris, Publications orientalistes de France, 1982.

Mryake Hitoshi 宮家準, Shugendō girei no kenkyū 修験道儀礼の研究 (Étude sur les rites de la voie de l'ascèse), Tōkyō, Shunjūsha 春秋社, 1985.

MiYaKe Hitoshi 宮家準, Shugendō to Nihon shükyō 修験道と日本宗教 (Le shugendō et la religion japonaise), Tōkyō, Shunjūsha, 1996. 
92 Cahiers d'études japonaises $n^{\circ} 21$

MrYaKe Hitoshi 宮家準, Nihon shükyō-shi ni okeru shugendō no ichi 日本宗教 史における修験道の一 (Une des voies de l'ascèse dans l'histoire des religions japonaises), Tōkyō, université de Waseda, 1987.

MORIGUCHI Tari 森口田理, Iwate no minzoku geinō yamabushi kagura hen 岩手 の民俗芸能山伏神楽編 (Recueil sur les kagura yamabushi, spectacles populaires folkloriques du département d'Iwate), Iwate, Iwate-ken kyōiku iinkai 岩 手県教育委員会, 1963 .

ORIKUCHI Shinobu 折口信夫, 《Kadomatsu no hanashi »門松のはなし (Discours sur le pin du seuil), in Zensh $\bar{u}$, kodai no kenkyū 全集 古代の研究 (OEuvres complètes, Recherches sur l'Ancien Temps), vol. 17, Tōkyō, Chūkō bunko 中公文庫, 1975, p. 463-469.

Philıppi Donald L. (dir.), Kojiki, Tōkyō, University of Tōkyō Press, 1968.

Plutschow, Herbert E., «Chaos and Cosmos » Rituel in Early and Medieval Japanese Literature, Leiden, E. J. Brill, 1990.

Renondeau Gaston, Le Shugendō : histoire, doctrine et rites des anachorètes dits Yamabushi, Paris, Imprimerie Nationale, 1965.

SASAKI Naoto 佐々木直人, OTtsugunai no yamabushi kagura 大償の山伏神 楽 (Le kagura yamabushi d'Ōtsugunai), vol. 1, édition à compte d'auteur de Sasaki Yutaka, 1953.

Sugawara Seiichirō 菅原盛一郎, Nihon no geinō, Hayachine-ryū yamabushi kagura 日本之芸能 早地峰流山伏神楽 (Spectacles populaires japonais, kagura yamabushi, les courants de Hayachine), Iwate, Tōwa-chō kyōiku iinkai 東和町教育委員会, 1979 [1969].

Résumé : Cet article est un extrait de la thèse de Catherine Delpuech qui paraîtra sous forme de livre. Il aborde la tradition des divertissements divins ou kagura qui perdure dans la région du mont Hayachine (Nord-Est du Japon) 
depuis au moins 500 ans. L'auteure fait une description commentée d'un rituel de douze danses, nommé journée classique, et objet de nombreuses exégèses savantes mises ici en opposition avec la réalité des représentations actuelles.

Mots-clés : danse, musique, religion, theater, Hayachine, Kagura, yamabushi, kami

Abstract: This article is an excerpt from Catherine Delpuech's thesis, which will be published as a book. It discusses the tradition of divine entertainments known as kagura, which has been performed in the region of Mt Hayachine (North-East of Japan) for at least 500 years. The author makes a narrated description of a ritual of 12 dances she calls "the classic day", which has been the object of much scholarly interest, and compares it with the reality of its current performances.

Keywords: Dance, Music, Religion, Theatre, Hayachine, Kagura, Yamabushi, Kami

キーワード : 踊り, 音楽, 宗教, 劇, 早池峰, 神楽, 山伏, 神 